\title{
Ação antioxidante de chás e condimentos de grande consumo
}

\section{no Brasil}

\author{
Selene M. de Morais, * Eveline S. B. Cavalcanti, Sônia Maria O. Costa, Liza A. Aguiar \\ Laboratório de Química de Produtos Naturais, Curso de Química, Universidade Estadual do Ceará, Av. \\ Paranjana, 1700, 60740-000 Fortaleza-CE, Brasil
}

\begin{abstract}
RESUMO: Antioxidantes são compostos que atuam inibindo e/ou diminuindo os efeitos desencadeados pelos radicais livres e compostos oxidantes. Os chás são bebidas populares e fontes significativas de compostos fenólicos, sendo considerados importantes integrantes das dietas devido às suas propriedades antioxidantes. O presente trabalho teve como objetivo avaliar a ação antioxidante de uma variedade de chás e condimentos mais consumidos no Brasil. Os chás analisados foram das plantas: Pneumus boldus Mold., Matricaria recutita L., Cymbopogon citratus (DC) Stapf, Baccharis trimera (Less.) DC, Camelia sinensis (L) O. Kuntze (fermentado), Camelia sinensis (não-fermentado), Lippia alba N.E. Brown, Mentha arvensis L., e Pyrus malus L. Os condimentos analisados foram: Eugenia aromatica Baill, Cinnamonum zeylanicum Blume, Laurus nobilis L. e Origanum vulgare L. O método utilizado para avaliar a ação antioxidante foi o da atividade seqüestradora de radicais livres DPPH em solução metanólica. Todas as amostras analisadas demonstraram atividade em suas diferentes concentrações. Camelia sinensis (nãofermentada) foi a mais ativa com $\mathrm{CI}_{50}=0,14 \mathrm{mg} / \mathrm{mL}$, cujos principais compostos antioxidantes são epigalocatequinas. Os condimentos mais ativos foram Cinnamonum zeylanicum $\left(\mathrm{CI}_{50}=0,37\right.$ $\mathrm{mg} / \mathrm{mL})$, Eugenia aromatica Baill $\left(\mathrm{CI}_{50}=0,46 \mathrm{mg} / \mathrm{mL}\right)$ e Laurus nobilis $\left(\mathrm{CI}_{50}=0,76 \mathrm{mg} / \mathrm{mL}\right)$, cujo principal antioxidante relatado foi o eugenol.
\end{abstract}

Unitermos: Atividade antioxidante, chás, condimentos.

\begin{abstract}
Antioxidant action of teas and seasonings more consumed in Brazil”. Antioxidants are compounds that remove free-radicals or minimize their availability to generate oxidative stress. Teas are popular beverages providing a significant source of phenolic compounds, important components of the human diet due to their antioxidant properties. The present work had the objective of evaluate the antioxidant action of teas and seasonings more consumed in Brazil. The analysed teas were from the plants: Pneumus boldus Mold., Matricaria recutita L., Cymbopogon citratus (DC) Stapf, Baccharis trimera (Less.) DC, Camelia sinensis (L) O. Kuntze (fermented), Camelia sinensis (not-fermented), Lippia alba N.E. Brown, Mentha arvensis L. and Pyrus malus L. The studied seasonings were: Eugenia aromatica Baill, Cinnamonum zeylanicum Blume, Laurus nobilis L. and Origanum vulgare L. The antioxidant activity was measured by the DPPH free radical scavenging method. All analysed samples demonstrated antioxidant activities. Camelia sinensis (not-fermented) $\left(\mathrm{IC}_{50}=0.14 \mathrm{mg} / \mathrm{mL}\right.$ ), was the most active and active principles are epicalocatechins. The stronger antioxidant seasonings were Cinnamonum zeylanicum $\left(\mathrm{IC}_{50}=0.37 \mathrm{mg} / \mathrm{mL}\right)$, Eugenia aromatica Baill $\left(\mathrm{IC}_{50}=0.46 \mathrm{mg} /\right.$ $\mathrm{mL}$ and Laurus nobilis $\left(\mathrm{IC}_{50}=0.76 \mathrm{mg} / \mathrm{mL}\right)$, which presented as main antioxidant component eugenol.
\end{abstract}

Keywords: Antioxidant action, teas, seasonings.

\section{INTRODUÇÃO}

Os antioxidantes podem ser definidos como substâncias capazes de retardar ou inibir a oxidação de substratos oxidáveis, podendo estes serem enzimáticos ou não enzimáticos, tais como: $\alpha$-tocoferol (vitamina E), $\beta$-caroteno, ascorbato (vitamina C) e os compostos fenólicos (flavonóides) (Haliwell, 2001; Sousa et al., 2007). O consumo de antioxidantes naturais, como os compostos fenólicos presentes na maioria das plantas que inibem a formação de radicais livres, também chamados de substâncias reativas, tem sido associado a uma menor incidência de doenças relacionadas com o estresse oxidativo (Droge, 2002). O estresse oxidativo ocorre como um desequilíbrio entre o balanço pró-oxidante/antioxidante, em favor da situação próoxidante, promovendo um dano potencial. O dano oxidativo que as biomoléculas sofrem está relacionado com as patologias de um grande número de doenças crônicas, incluindo doenças cardiovasculares, câncer 
e doenças neurodegenerativas (Wiseman et al., 2001; Liao et al., 2001; Javanmardi et al., 2002; Lu \& Yeap, 2002; Kim et al., 2003; Mendel \& Youdim, 2004).

Nos últimos anos, têm-se investigado os efeitos dos antioxidantes em relação às enfermidades, principalmente nos paises desenvolvidos do ocidente. As pesquisas têm tentado explicar os benefícios dos antioxidantes nas enfermidades cardiovasculares, em numerosos tipos de câncer, na AIDS, e inclusive em outros diretamente associados com o processo de envelhecimento, como o das cataratas, doença de Alzheimer e outras alterações do sistema nervoso (Cai et al., 2004; Netzel et al., 2007; Jayaprakasha et al., 2007).

Tanto óleos essenciais (Baratta et al., 1998; Ruberto \& Baratta, 2000; Morais et al., 2006; Souza et al., 2007) como componentes não voláteis (extratos), extraídos de plantas (Weng \& Wang, 2000; Fenglin et al., 2004; Miliauskas et al., 2004; Andrade et al., 2007; Sousa et al., 2007; Balestrin et al., 2008; Nunes et al., 2008), têm sido estudados quanto à avaliação do seu potencial antioxidante, demonstrando alta eficiência.

As atividades antioxidantes dos fenóis são devidas às suas propriedades de oxirredução, que permitem agirem como agentes redutores, doadores de hidrogênio e eliminadores de oxigênio singlete (Sousa et al., 2007; Guerra, 2001).

Atribui-se o efeito antioxidante das plantas aromáticas à presença de grupamentos hidroxilas em seus compostos fenólicos (Shahidi et al., 1992). Das classes de compostos químicos que são responsáveis pela inibição da peroxidação lipídica, presentes na maioria dos produtos naturais, estão os polifenóis, flavonóides e taninos. Os flavonóides são também responsáveis pela inibição de oxidação do ácido linolêico, oxidação de LDL, peroxidação de fosfolipídeos da membrana, peroxidação lipídica microssomal e mitocondrial, peroxidação de eritrócitos e fotoxidação e peroxidação de cloroplastos (Anila \& Vijayalakshmi, 2003; Santanam et al., 2004). Nas pesquisas bibliográficas realizadas por Heim et al., (2002) são citadas diversas ações de flavonóides como antioxidantes, relacionando a química, metabolismo e a relação estrutura atividade.

O chá é uma das bebidas mais consumidas e mais antigas do mundo, sendo na literatura referido como uma das melhores fontes de compostos fenólicos (Lima et al., 2004). Os primeiros relatos de seu uso datam do século 27 a.C., sendo considerado como uma das mais antigas bebidas produzidas por via biotecnológica e praticada pelo ser humano.

Os chás têm atraído muita atenção nos últimos anos devido a sua capacidade antioxidante e sua abundância na dieta de milhares de pessoas em todo o mundo. São ricos em catequinas, flavonóides que apresentam propriedades biológicas como atividade antioxidante e seqüestradoras de radicais livres. Os chás ingeridos na forma de infusão contribuem para a extração dos compostos fenólicos, considerados benéficos à saúde (Higdon \& Frei, 2003; Mendel \& Youdim, 2004; Bunkova et al., 2005).

Os condimentos são mundialmente utilizados para aumentar e/ou acrescentar sabor ao alimento, e, secundariamente, com finalidade de conservação, devido às suas propriedades antimicrobianas e antioxidantes. Os condimentos, por exemplo, da família Lamiaceae têm sido extensivamente estudados devido ao caráter antioxidante de seus compostos fenólicos, porém apenas o alecrim tem sido mais amplamente utilizado em produtos alimentícios. Mariutti \& Bragagnolo (2007) em sua revisão sobre os principais antioxidantes naturais da família Laminaceae abordam os compostos responsáveis pela atividade antioxidante e suas aplicações em produtos alimentícios.

Vários estudos relatam a presença de antioxidantes em chás, mas a metodologia utiliza extratos obtidos por solventes orgânicos das folhas secas. Há poucos relatos sobre os compostos fenólicos e atividade antioxidante em infusões de ervas. Assim como os chás, várias espécies de condimentos demonstram interesse quanto à avaliação do potencial antioxidante devido seu uso comum na culinária brasileira. Neste trabalho foram avaliados, quanto a sua atividade antioxidante, vários chás e condimentos que são largamente consumidos pela população brasileira para os mais diversos fins (Tabela 1).

\section{MATERIAL E MÉTODOS}

\section{Material vegetal}

As amostras de chás e de condimentos utilizadas foram obtidas em redes de supermercado da cidade de Fortaleza (Ceará). Os chás utilizados foram: boldo (Peumus boldus Mold.,), camomila (Matricaria recutita L.), capim santo (Cymbopogon citratus (DC) Stapf), carqueja (Baccharis trimera (Less.) DC), chá preto (Camelia sinensis (fermentado)), chá verde (Camelia sinensis (L) O. Kuntze (não-fermentado)), cidreira (Lippia alba N.E. Brown), hortelã (Mentha arvensis L.), e maçã (Pyrus malus L.). Os condimentos utilizados foram: cravo (Eugenia aromatica Baill), canela (Cinnamonum zeylanicum Blume), louro (Laurus nobilis L.) e orégano (Origanum vulgare L.).

\section{Avaliação da ação antioxidante}

Para avaliação da atividade antioxidante foi utilizado o método seqüestrador de radicais livres DPPH (1,1-difenil-2-picril-hidrazila) descrito por Yepez et al. (2002) e Fenglin et al., (2004) com pequenas modificações como segue: em um tubo de ensaio, foram colocados 2,0 mL de uma solução metanólica de radical livre DPPH 6,5 x 10-5 M. Em seguida foram adicionados ao tubo $2,0 \mathrm{~mL}$ da solução metanólica do extrato $(0,1 \mathrm{~g} /$ 
Tabela 1. Chás e condimentos: constituintes químicos, usos populares e $\mathrm{CI}_{50}$ *

\begin{tabular}{|c|c|c|c|c|c|}
\hline Nome vulgar & Nome científico & Composição química & Uso indicado & $\mathrm{CI}_{50}$ & Referência \\
\hline Boldo & $\begin{array}{l}\text { Pneumus boldus } \\
\text { Mold. }\end{array}$ & $\begin{array}{l}\text { Alcalóides (boldina) com } \\
\text { atividade antioxidante), } \\
\text { taninos, esteróides, ácidos } \\
\text { graxos, flavonóides } \\
\text { (quercetina e canferol } \\
\text { e derivados flavônicos } \\
\text { (boldosídio, peumosídio), } \\
\text { eugenol. }\end{array}$ & $\begin{array}{l}\text { Má digestão, distúrbios } \\
\text { hepáticos, manifestações } \\
\text { reumáticas e inflamações do } \\
\text { trato urinário }\end{array}$ & $\begin{array}{l}2,04 \mathrm{mg} / \\
\mathrm{mL}\end{array}$ & $\begin{array}{l}\text { Sousa et } \\
\text { al., 2004; } \\
\text { Santanam } \\
\text { et al., 2004; } \\
\text { Matsubara, } \\
2006 \text {. }\end{array}$ \\
\hline Camomila & Matricaria recutita L. & $\begin{array}{l}\text { Óleo essencial (camazuleno). } \\
\text { Ácidos fenólicos, esteróides, } \\
\text { taninos, cumarinas e } \\
\text { flavonóides (quercetina). }\end{array}$ & $\begin{array}{l}\text { Ação interna: antiespasmódica, } \\
\text { calmante, atenuante de gases } \\
\text { no estômago. }\end{array}$ & $\begin{array}{l}47,41 \mathrm{mg} / \\
\mathrm{mL}\end{array}$ & $\begin{array}{l}\text { Sousa et } \\
\text { al., 2004; } \\
\text { Matsubara, } \\
2006\end{array}$ \\
\hline Capim Santo & $\begin{array}{l}\text { Cymbopogon citratus } \\
\text { (DC) Stapf }\end{array}$ & $\begin{array}{l}\text { Óleo essencial contém } \\
\text { principalmente citral, } \\
\text { mentol, limoneno e linalol. } \\
\text { Flavonóides e alcalóides. }\end{array}$ & $\begin{array}{l}\text { Calmante, sedativo, problemas } \\
\text { gastrointestinais, repelente de } \\
\text { insetos, tratamento de diabetes } \\
\text { e úlcera. }\end{array}$ & $\begin{array}{l}17,36 \mathrm{mg} / \\
\mathrm{mL}\end{array}$ & $\begin{array}{l}\text { Sousa et al., } \\
\text { 2004; Melo et } \\
\text { al., } 2001 .\end{array}$ \\
\hline Carqueja & $\begin{array}{l}\text { Baccharis trimera } \\
\text { (Less.) DC }\end{array}$ & $\begin{array}{l}\text { Óleo essencial (pineno, } \\
\text { carquejol, acetato de } \\
\text { carquejilo e sesquiterpenos). }\end{array}$ & $\begin{array}{l}\text { Exerce ação benéfica sobre o } \\
\text { fígado e intestinos. }\end{array}$ & $\begin{array}{l}44,07 \mathrm{mg} / \\
\mathrm{mL}\end{array}$ & $\begin{array}{l}\text { Sousa et al., } \\
2004 .\end{array}$ \\
\hline Chá Verde & $\begin{array}{l}\text { Camelia sinensis }(\mathrm{L}) \\
\text { O. Kuntze } \\
\begin{array}{l}\text { Camelia sinensis } \\
\text { (fermentada) }\end{array}\end{array}$ & $\begin{array}{l}\text { Vitaminas do complexo B e } \\
\text { C; Ácidos fenolcarboxílicos; } \\
\text { Taninos antioxidantes - } \\
\text { galato de epigalocatequina; } \\
\text { catequinas (epicatequina, } \\
\text { epigalocatequina, galato- } \\
\text { 3-epicatequina e galato-3- } \\
\text { epigalocatequina), Bases } \\
\text { púricas (cafeína, teofilina, } \\
\text { treobomina). }\end{array}$ & $\begin{array}{l}\text { Diurético adstringente, } \\
\text { antioxidante, astenia física } \\
\text { e psíquica, tratamento de } \\
\text { diarréia } \\
\text { Asma, prevenção de } \\
\text { arteriosclerose, anticâncer. }\end{array}$ & $\begin{array}{l}0,14 \mathrm{mg} / \\
\mathrm{mL} \\
\\
\\
0,96 \quad \mathrm{mg} / \\
\mathrm{mL}\end{array}$ & $\begin{array}{l}\text { Sousa et } \\
\text { al., 2004; } \\
\text { Luypaert et al., } \\
\text { 2003; } \\
\text { Ferrara et al., } \\
2001 \text {; } \\
\text { Schmitz et al., } \\
2005 \text {. }\end{array}$ \\
\hline Cidreira & $\begin{array}{l}\text { Lippia alba } \\
\text { N.E.Brown. }\end{array}$ & $\begin{array}{l}\text { Limoneno, carvona, citral, } \\
\beta \text {-mirceno, heterosídeo } \\
\text { flavonoídico. }\end{array}$ & $\begin{array}{l}\text { Tranqüilizante e auxiliar em } \\
\text { casos de insônia e tratamento } \\
\text { de distúrbios gastrointestinais. }\end{array}$ & $\begin{array}{l}27,29 \mathrm{mg} / \\
\mathrm{mL}\end{array}$ & $\begin{array}{l}\text { Matos et al., } \\
2001 .\end{array}$ \\
\hline Hortelã & Mentha arvensis L. & $\begin{array}{l}\text { Óleo essencial (mentol, } \\
\text { isomentona), ácidos } \\
\text { orgânicos, sitosterol. }\end{array}$ & $\begin{array}{l}\text { Combate a contração muscular } \\
\text { brusca (espasmolítica). } \\
\text { Nas afecções estomacais e } \\
\text { intestinais. }\end{array}$ & $\begin{array}{l}17,40 \mathrm{mg} / \\
\mathrm{mL}\end{array}$ & $\begin{array}{l}\text { Sousa et al., } \\
2004 .\end{array}$ \\
\hline Maçã & Pyrus malus L. & $\begin{array}{l}\text { Ácidos málico e cítrico, } \\
\text { enzimas, sais minerais } \\
\text { e vitaminas (tiamina, } \\
\text { riboflavina, ácido ascórbico) }\end{array}$ & $\begin{array}{l}\text { Regulador das funções } \\
\text { intestinais; combate artrite, } \\
\text { reumatismo, cálculos urinários } \\
\text { e diminui o colesterol. }\end{array}$ & $\begin{array}{l}19,27 \mathrm{mg} / \\
\mathrm{mL}\end{array}$ & $\begin{array}{l}\text { Sousa et al., } \\
2004 .\end{array}$ \\
\hline Canela & $\begin{array}{l}\text { Cinnamonum } \\
\text { zeylanicum } \\
\text { Blume. }\end{array}$ & $\begin{array}{l}\text { Óleo essencial (eugenol, alfa- } \\
\text { felandreno, alfa-terpineol, } \\
\text { alfa-pineno, aldeído } \\
\text { cinâmico). }\end{array}$ & $\begin{array}{l}\text { Uso como condimentos e } \\
\text { aromatizantes; estomáquico } \\
\text { e sudorífico; propriedades } \\
\text { antibacterianas e antifúngicas. }\end{array}$ & $\begin{array}{l}0,37 \mathrm{mg} / \\
\mathrm{mL}\end{array}$ & $\begin{array}{l}\text { Sousa et al., } \\
2004 .\end{array}$ \\
\hline Cravo & $\begin{array}{l}\text { Eugenia aromatica } \\
\text { Baill. }\end{array}$ & $\begin{array}{l}\text { Óleo essencial (eugenol, } \beta \text { - } \\
\text { cariofileno, acetil-eugenol, } \\
\text { metil-eugenol, oxido de } \\
\text { cariofileno, chavicol). }\end{array}$ & $\begin{array}{l}\text { Uso como condimentos e } \\
\text { aromatizantes; estimulante das } \\
\text { funções digestivas. }\end{array}$ & $\begin{array}{l}0,46 \mathrm{mg} / \\
\mathrm{mL}\end{array}$ & $\begin{array}{l}\text { Sousa et al., } \\
2004 .\end{array}$ \\
\hline Louro & Laurus nobilis L. & $\begin{array}{l}\text { Óleo essencial (eugenol, } \\
\text { metileugenol, pineno). }\end{array}$ & $\begin{array}{l}\text { Uso como condimentos } \\
\text { e aromatizantes, ação } \\
\text { carminativa e digestiva, } \\
\text { hemorróidas, reumatismo, } \\
\text { contusões e anticonvulsivante. }\end{array}$ & $\begin{array}{l}0,76 \mathrm{mg} / \\
\mathrm{mL}\end{array}$ & $\begin{array}{l}\text { Sayyah. et al., } \\
2002 .\end{array}$ \\
\hline Orégano & Origanum vulgare L. & $\begin{array}{l}\text { Óleo essencial (Timol) e } \\
\text { flavanóides. }\end{array}$ & $\begin{array}{l}\text { Atividade anti-oxidante, anti- } \\
\text { microbianas contra bactérias } \\
\text { e o utros patógenos presentes } \\
\text { nos alimentos, Uso como } \\
\text { condimentos e aromatizantes. }\end{array}$ & $\begin{array}{l}1,08 \mathrm{mg} / \\
\mathrm{mL}\end{array}$ & $\begin{array}{l}\text { Cervato et al., } \\
2000 .\end{array}$ \\
\hline
\end{tabular}

* $\mathrm{CI}_{50}=$ concentração do da amostra que inibe em $50 \%$ o teor do radical livre DPPH.

$\mathrm{mL}$ ) a ser testado. Após 20 minutos de reação, foi medida a absorbância em um espectrofotômetro Spekol 1100 no comprimento de onda de $517 \mathrm{~nm}$. O procedimento foi feito em diferentes concentrações para cada uma das amostras pesquisadas.

A atividade antioxidante dos extratos desses chás foi determinada através da sua capacidade de seqüestrar o radical DPPH. O método é baseado 
<smiles>O=C(O[C@@H]1Cc2c(O)cc(O)cc2O[C@@H]1c1cc(O)c(O)c(O)c1)c1cc(O)c(O)c(O)c1</smiles>

Galato-3 - epigalocatequina

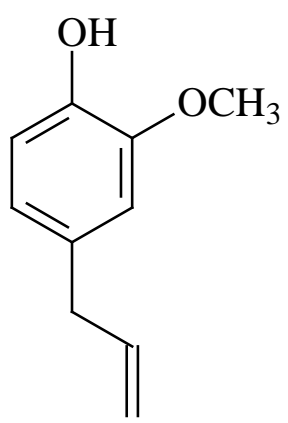

Eugenol

Figura 1. Principais constituintes antioxidantes dos chás e condimentos de grande consumo pela população brasileira.

na variação da absorbância obtida por uma perda estequiométrica da cor da solução do radical na presença de substâncias antioxidantes presentes na amostra do extrato, para calcular o Índice de Varredura (IV) do $\mathrm{DPPH}$ em percentual foi usada a fórmula: $\mathrm{IV} \%=\left(\mathrm{A}_{\mathrm{DPPH}}\right.$ - A $/ A_{\text {DPPH }}$ ) x 100

Onde $A_{D P P H}$ é a absorbância inicial da solução de DPPH e A é a absorbância final, decorridos 20 minutos de reação. Para comparação, usou-se o Eugenol como padrão na proporção molar eugenol/DPPH de 0,5 $\mathrm{mg} / \mathrm{mL}$. Com os resultados dos IV\% obtidos na leitura da absorbância das diferentes concentrações de cada uma das amostras foi possível calcular o índice capaz de inibir 50\% dos radicais livres $\left(\mathrm{CI}_{50}\right)$ (Lee et al., 2003).

\section{RESULTADOS E DISCUSSÃO}

Na Tabela 1 encontram-se os dados obtidos dos testes da atividade antioxidante dos chás e condimentos comumente consumidos pela população brasileira, juntamente com a constituição química e uso popular de cada espécie estudada.

Os resultados variaram em função do tipo de chá e do condimento. Dentre as amostras analisadas, as que se mostraram mais eficientes frente aos radicais livres foram: chá verde (Camelia sinensis (não-fermentada)) com IC $_{50}$ de $0,14 \mathrm{mg} / \mathrm{mL}$, canela (Cinnamonum zeylanicum) com $\mathrm{CI}_{50}$ de $0,37 \mathrm{mg} / \mathrm{mL}$, cravo (Eugenia aromatica) com $\mathrm{CI}_{50}$ de $0,46 \mathrm{mg} / \mathrm{mL}$, louro (Laurus nobilis) com $\mathrm{CI}_{50}$ de $0,76 \mathrm{mg} / \mathrm{mL}$ e chá preto (Camelia sinensis (fermentada)) com $\mathrm{CI}_{50}$ de $0,96 \mathrm{mg} / \mathrm{mL}$.

$\mathrm{O}$ bom poder antioxidante dessas plantas pode ser explicado pela presença de substâncias capazes de inibir os radicais livres. Basicamente há três tipos de chás tendo como nome científico Camelia sinensis, que são mais consumidos mundialmente: chá verde (sem fermentação), Oolong (parcialmente fermentado) e chá preto (totalmente fermentado). Os constituintes mais importantes das folhas do chá de Camelia sinensis são compostos fenólicos, em especial taninos (ácido gálico) e flavonóides (quercetina, miricetina, canferol e catequinas) (Lima et al., 2004; Matsubara \& RodriguezAmaya, 2006; Bettelheim et al., 1995; Ferrara et al., 2001). De acordo com dados da literatura (Schmitz et al., 2005) as catequinas são polifenóis responsáveis pela cor e corpo do chá, que correspondem a $26,7 \%$ dos compostos presentes na espécie Camelia sinensis (chá verde), dos quais $11 \%$ são constituídos de galato-3epigalocatequina (EGCG), 10\% galato-3-epicatequina (EGC), 2,5\% epicatequina (EC) e $2 \%$ epigalocatequina (ECG). Neste estudo, a substância que apresentou maior atividade antioxidante foi a EGCG (Figura 1).

Os condimentos analisados que obtiveram os melhores $\mathrm{CI}_{50}$, canela (Cinnamonum zeylanicum), cravo (Eugenia aromatica Baill), e louro (Laurus nobilis) têm em sua constituição um forte antioxidante como o eugenol, um composto fenólico (4-alil-2-metoxifenol) (Figura 1), cuja eficácia já foi comprovada tanto in vitro como in vivo (Ito et al., 2005). Todos os materiais testados demonstraram ação antioxidante em suas diferentes concentrações.

O bom desempenho desses produtos deve-se à presença de flavonóides, catequinas e outros compostos fenólicos já relatados na literatura como capazes de inibir os radicais livres presentes no organismo. As variedades de chás e condimentos testadas possuem um bom potencial antioxidante quando comparadas com o eugenol cuja atividade já é comprovada.

Portanto o conhecimento das propriedades antioxidantes dos produtos de nosso consumo diário pode direcionar a população apropriadamente na escolha de um produto com maior poder medicinal. Os resultados descritos neste trabalho estimulam a continuidade da pesquisa para avaliar o poder antioxidante de substãncias isoladas das espécies estudadas. 


\section{AGRADECIMENTOS}

Os autores agradecem ao Programa Institucional de Bolsas de Iniciação Científica - PIBIC/CNPq da Universidade Estadual do Ceará e a FUNCAP, pelo apoio financeiro para o desenvolvimento deste trabalho.

\section{REFERÊNCIAS}

Andrade CA, Costa CK, Bora K, Miguel MD, Miguel OG, Kerber VA 2007. Determinação do conteúdo fenólico e avaliação da atividade antioxidante de Acacia podalyriifolia A. Cunn. ex G. Don, Leguminosaemimosoideae. Rev Bras Farmacogn 17: 231-235.

Anila L, Vijayalakshmi NR 2003. Antioxidant action of flavonoids from Mangifera indica and Emblica officinalis in hypercholesterolemic rats. Food Chem 83: 569-574.

Balestrin L, Dias JFG, Miguel OG, Dall’Stella DSG, Miguel MD 2008. Contribuição ao estudo fitoquímico de Dorstenia multiformis Miquel (Moraceae) com abordagem em atividade antioxidante. Rev Bras Farmacogn 18: 230-235.

Baratta MT, Dorman HJD, Deans SG, Biondi D, Ruberto G 1998. Chemical composition, antimicrobial and antioxidative activity of laurel, sage, rosemary oregano and coriander essential oils. J Essent Oil Res 10: 618-627.

Bettelheim FA, Landesberg J, Lee J 1995. Laboratory Experiments for General, Organic and Biochemistry. Saunders College Publishing, 552p.

Bunkova R, Marova I, Nemec M 2005. Antimutagenic properties of green tea. Plant Foods Hum Nutr 60: 25-29.

Cai Y, Luo Q, Sun M, Corke H 2004. Antioxidant activity and phenolic compounds of 112 traditional Chinese medicinal plants associated with anticancer. Life Sci 74: 2157-2184.

Cervato G, Carabelli M, Gervasio S, Cittera A, Cazzola R, Cestaro B 2000. Antioxidant properties of oregano (Origanum vulgare) leaf extracts. J Food Biochem 24: 453-465.

Droge W 2002. Free radicals in the physiological control of cell function. Physiol Rev 82: 47-95.

Fenglin H, Ruili L, Bao H, Liang M 2004. Free radical scavenging activity of extracts prepared from fresh leaves of selected Chinese medicinal plants. Fitoterapia 75: 14-23.

Ferrara L, Montesano D, Senatore A 2001. The distribution of minerals and flavonoids in the tea plant (Camellia sinensis). Farmaco 56: 397-401.

Guerra EJI 2001. Oxidative stress, diseases and antioxidant treatment. Ann Med Interne 18: 326.

Halliwell B 2001. Free radicals and other reactive species in disease. In: Encyclopedia of Life ciences. Nature Publishing Group, p. 1-7.

Heim, KE, Tagliaferro AR, Bobilya DJ 2002. Flavonoid antioxidants: chemistry, metabolism, and structure -activity relationships. Reviews: current topics. $J$ Nutr Biochem 13: 572-584.

Higdon JV, Frei B 2003. Tea catechins and polyphenols: health effects, metabolism, and antioxidant functions. Crit
Rev Food Sci Nutr 43: 89-143.

Ito M, Murakami K, Yoshino M 2005. Antioxidant action of eugenol compounds: role of metal ion in the inhibition of lipid peroxidation. Food Chem Toxicol 43: 461-466.

Javanmardi J, Khalighi A, Kashi A, Bais HP, Vivanco JM 2002. Chemical characterization of basil (Ocimum basiliam L.) found in local acessions and used in traditional medicines in Iran. J Agric Food Chem 50: 5878-5883.

Jayaprakasha GK, Mandadi, KK, Poulose SM, Jadegoud Y, Nagana Gowda GA, Patil BS 2007. Inhibition of colon cancer growth and antioxidant activity of bioactive compounds from Poncirus trifoliate (L.) Raf. Bioorg Med Chem 15: 4923-4932.

Kim DO, Jeong W, Lee CY 2003. Antioxidant capacity of phenolic phytochemicals from various cultivars of plums. Food Chem 81: 321-326.

Lee SE, Hwang HJ, Ha JS, Jeong HS, Jeong HK 2003. Screening medicinal plant extracts for antioxidant activity. Life Sci 73: 167-179.

Liao S, Kao YH, Hiipakka R 2001. Green tea: biochemical and biological basis for health benefits. Vitam Horm 62: 1-94.

Lima VLAG, Melo EA, Lima DESL 2004. Nota prévia: Teor de compostos fenólicos totais em chás brasileiros. Braz J Food Technol 7: 187-190.

Lu Y, Yeap FL 2002. Poliphenolics of Salvia - a review. Phytochemistry 59: 117-140.

Luypaert J, Zhang MH, Massart DL 2003. Feasibility study for the use of near infrared spectroscopy in the quantitative analysis of green tea, Camellia sinensis (L.). Anal Chim Acta 478: 303-312.

Mariutti LRB, Braganolo N 2007. Revisão: Antioxidantes naturais da família Lamiaceae - Aplicação em produtos alimentícios. Braz J Food Technol 10: 96103.

Matsubara S, Rodriguez-Amaya DB 2006. Conteúdo de mircetina, quercetina e kaempferol em chás comercializados no Brasil. Cienc Teconol Aliment 26: 380-385.

Melo SF, Soares SF, Costa RF, Silva CR, Oliveira MBN, Bezerra RJAC, Araújo AC, Bernardo Filho B 2001. Effect of Cymbopogon citratus, Maytenus ilicifolia and Baccharis genistelloides extracts against the stannous chloride oxidative damage in Escherichia colli. Mutat Res 496: 33-38.

Mendel S, Youdim MB 2004. Catechin polyphenols: neurodegeneration and neuroprotection in neurodegenerative diseases. Free Radic Biol Med 37: 304-317.

Miliauskas G, Venskutonis PR, Van Beek TA 2004. Screening of radical scavenging of some medicinal and aromatic plants extracts. Food Chem 85: 231-237.

Morais SM, Catunda-Jr EA, Silva ARA, Martins-Neto JS 2006. Atividade antioxidante de óleos essenciais de espécies de Croton do Nordeste do Brasil. Quim Nova 29: 907-910.

Netzel M, Netzel G, Kammerer DR, Schieber A, Carle R, Simons L, Bitsch I, Bitsch R, Konczak I 2007. Cancer cell antiproliferation activity and metabolism of black carrot anthocyanins. Innov Food Sci Emerg Technol 8: 365-372. 
Nunes XP, Mesquita RF, Silva DA, Lira DP, Costa VCO, Silva MVB, Xavier AL, Diniz MFFM, Agra MF 2008. Constituintes químicos, avaliação das atividades citotóxica e antioxidante de Mimosa paraibana Barneby (Mimosaceae). Rev Bras Farmacogn 18 (Supl): 718-723.

Ruberto G, Baratta MT 2000. Antioxidant activity of selected essential oil components in two lipid model systems. Food Chem 69: 167-174.Santanam N, Penumetcha M, Speisky H, Parthasarathy S 2004. A novel alkaloid antioxidant, boldine and synthetic antioxidant, reduced form of RU486, inhibit the oxidation of LDL in-vitro and atherosclerosis in vivo in LDLR mice. Atherosclerosis 173: 203-210.

Sayyah M, Valizadeh J, Kamalinejad M 2002. Anticonvulsant activity of the leaf essential oil of Laurus nobilis, against pentyllenetetrazole-and maximal electroshock-induced seizures. Phytomedicine 9: 212-216.

Schahidi F, Janitha PK, Wanasundra PD 1992. Phenolic antioxidants. Crit Rev Food Sci Nutr 32: 67-103.

Schmitz W, Saito AY, Estevão D, Saridakis HO 2005. O chá verde e suas ações como quimioprotetor. Semin Cienc Biol Saude 26: 119-130.

Sousa MP, Matos MEO, Matos FJA, Machado MIL, Craveiro AA 2004. Constituintes químicos ativos e propriedades biológicas de Plantas Medicinais Brasileiras, Ed. UFC, 445p.

Sousa CM, Silva HRE, Vieira-Jr GM, Ayres MCC, Costa CLS, Araújo DS, Cavalcante LCD, Barros EDS, Araújo PBM, Brandão MS, Chaves MH 2007. Fenóis totais e atividade antioxidante de cinco plantas medicinais. Quim Nova 30: 351-355.

Souza TJT, Apel MA, Bordignon S, Matzenbacher NI, Zuanazzi JAS, Henriques AT 2007. Composição química e atividade antioxidante do óleo volátil de Eupatorium polystachyum DC. Rev Bras Farmacogn 17: 368-372.

Weng XC, Wang W 2000. Antioxidant activity of compounds isolated from Salvia plebeian. Food Chem 71: 489493.

Wiseman S, Waterhouse A, Korver O 2001. The health effects of tea and tea components: Opportunities for standardizing research methods. Crit Rev Food Sci Nutr 41: 387-412.

Yepez B, Espinosa M, López S, Bolaños G 2002. Producing antioxidant fractions from herbaceous matrices by supercritical fluid extraction. Fluid Phase Equil 194: 879-884. 